\title{
Particle and Heat Transport in a Dense Wall- Confined MTF Plasma (Theory and Simulations)
}

D.D. Ryutov, D.C. Barnes, B.S. Bauer, J.H. Hammer, C.W. Hartman, R.C. Kirkpatrick, I.R. Lindemuth, V. Makhin, P.B. Parks, D.B. Reisman, P.T. Sheehey, R.R. Siemon

This article was submitted to $19^{\text {th }}$ International Atomic Energy Agency Fusion Energy Conference, Lyon, France, October 14-19, U.S. Department of Energy 2002

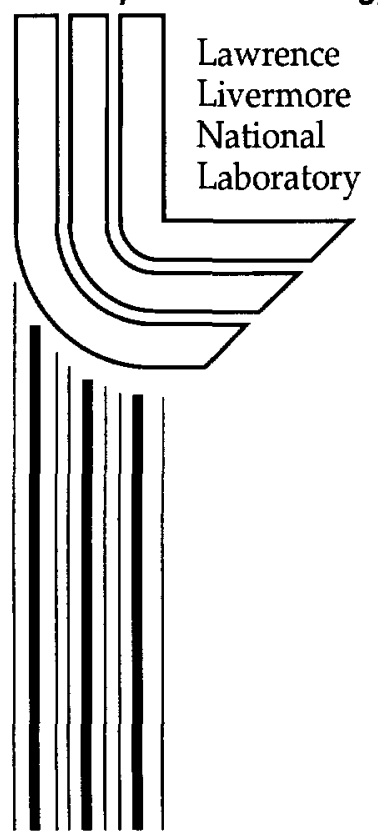

\section{September 25, 2002}




\section{DISCLAIMER}

This document was prepared as an account of work sponsored by an agency of the United States Government. Neither the United States Government nor the University of California nor any of their employees, makes any warranty, express or implied, or assumes any legal liability or responsibility for the accuracy, completeness, or usefulness of any information, apparatus, product, or process disclosed, or represents that its use would not infringe privately owned rights. Reference herein to any specific commercial product, process, or service by trade name, trademark, manufacturer, or otherwise, does not necessarily constitute or imply its endorsement, recommendation, or favoring by the United States Government or the University of California. The views and opinions of authors expressed herein do not necessarily state or reflect those of the United States Government or the University of California, and shall not be used for advertising or product endorsement purposes.

This is a preprint of a paper intended for publication in a journal or proceedings. Since changes may be made before publication, this preprint is made available with the understanding that it will not be cited or reproduced without the permission of the author.

This work was performed under the auspices of the United States Department of Energy by the University of California, Lawrence Livermore National Laboratory under contract No. W-7405-Eng-48.

This report has been reproduced directly from the best available copy.

Available electronically at http://www.doc.gov/bridge

Available for a processing fee to U.S. Department of Energy

And its contractors in paper from

U.S. Department of Energy

Office of Scientific and Technical Information

P.O. Box 62

Oak Ridge, TN 37831-0062

Telephone: (865) 576-8401

Facsimile: (865) 576-5728

E-mail: reports@adonis.osti.gov

Available for the sale to the public from

U.S. Department of Commerce

National Technical Information Service

5285 Port Royal Road

Springfield, VA 22161

Telephone: (800) 553-6847

Facsimile: (703) 605-6900

E-mail: orders@ntis.fedworld.gov

Online ordering: $\underline{\mathrm{http}: / / \text { www.ntis.gov/ordering.htm }}$

\section{$\mathrm{OR}$}

Lawrence Livermore National Laboratory

Technical Information Department's Digital Library

http://www.llnl.gov/tid/Library.html 


\title{
Particle and Heat Transport in a Dense Wall-Confined MTF Plasma (Theory and Simulations)
}

D.D. Ryutov' (ryutov1@llnl.gov), D.C. Barnes², B.S. Bauer ${ }^{3}$, J.H. Hammer', C.W. Hartman ${ }^{1}$, R.C. Kirkpatrick $^{2}$, I.R. Lindemuth ${ }^{2}$, V. Makhin ${ }^{3}$, P. B. Parks ${ }^{4}$, D.B. Reisman ${ }^{1}$, P.T. Sheehey ${ }^{2}$, R.E. Siemon ${ }^{3}$ ('Lawrence Livermore National Laboratory, USA; ${ }^{2}$ Los Alamos National Laboratory, USA; ${ }^{3}$ University of Nevada at Reno, USA; ${ }^{4}$ General Atomics, San Diego, USA)

\begin{abstract}
Plasma beta in Magnetized Target Fusion (MTF) systems is sometimes much greater than 1, and the plasma may be in direct contact with the imploding liner. Plasma processes are strongly dominated by interparticle collisions. Under such conditions, the plasma microturbulence, behavior of alpha particles, and plasma equilibria are very different from conventional fusion systems. The present paper contains the most comprehensive analysis of the corresponding phenomena to date. 2D numerical simulations of plasma convection in the targets of a diffuse pinch type demonstrate an onset of convection in this configuration.
\end{abstract}

\section{Introduction}

Magnetized target fusion is based on the quasi-adiabatic compression of a dense magnetized plasma by an imploding liner (see [1-3] and refs. therein). Possible plasma configurations include field reversed configuration (FRC), spheromaks, diffuse pinches, mirrors, and others (see [4] for more detail). The relative compactness of MTF systems, combined with the relative simplicity of the drivers allow the development of an attractive fusion reactor [3]. Plasma $\beta$ in MTF systems is large, sometimes significantly exceeding unity. In some versions of MTF systems (e.g., [2]) the plasma is supposed to be in direct contact with the imploding liner (so called wall confinement, [5]). The present paper contains the most comprehensive analysis of the corresponding phenomena to date. Another set of issues, mostly related to gross equilibrium and stability of one of the favorite MTF configurations - the FRC - will be covered in [6]. For most recent published results related to this latter set of problems see [7-9]. Key parameters of the systems which we will discuss below, are provided in TABLE I.

TABLE I. PARAMETERS OF TYPICAL MTF PLASMAS

\begin{tabular}{|c|c|c|c|c|c|c|c|c|c|}
\hline & $n, \mathrm{~cm}^{-3}$ & $T, \mathrm{eV}$ & $a, \mathrm{~cm}$ & $\beta$ & $E^{a}$ & $\varepsilon$ & $\mu$ & $M o$ & $s$ \\
\hline 1 & $10^{19}$ & $10^{3}$ & 0.3 & 3 & 5 & 0.05 & 0.2 & 45 & 20 \\
\hline 2 & $10^{21}$ & $5 \cdot 10^{3}$ & 0.1 & 10 & 5 & 0.021 & 0.17 & 44 & 42 \\
\hline 3 & $10^{17}$ & 150 & 3 & 2 & 10 & 0.009 & 0.023 & 25 & 25 \\
\hline 4 & $10^{15}$ & 100 & 0.5 & 0.2 & 20 & 1.47 & 0.3 & 1.7 & 4 \\
\hline
\end{tabular}

${ }^{a)} \mathrm{E}$ is the ratio of the plasma length to the plasma radius ("elongation").

1 - A compact, high-density MTF target half-way through the implosion; 2 - the same target near the point of the maximum compression [2]. 3 - FRC experiment at LANL [10]: the plasma parameters taken halfway between the $O$ point and the separatrix. 4 - Plroposed inverse-pinch experiment at UN Reno; the value for $a$ is radial length-scale of the plasma compressed against the external wall, the wall radius is $R=10 \mathrm{~cm}$; in this case we define $E$ as $E=R / a$. The parameters $\varepsilon, \mu, M_{0}$, and $s$ are defined in the next section.

\section{Plasma microinstabilities}

Because of a high plasma density, the MTF system may show a reasonable performance even if the cross-field transport coefficients are comparable with the Bohm diffusion coefficient, $D_{B o h m}=c T / 16 e B$ [2]. However, Ref. [11] where a high-beta plasma confinement was analyzed for the collisionless plasmas, predicts transport coefficients as high as $10 D_{\text {Bohm }}$ which are unacceptably high. It was pointed out in [12] that the results of [11] cannot be applied to MTF plasmas because of their high collisionality. Here we present a summary of a systematic assessment of a plasma transport in MTF systems, within the mixing-length theory (the same as in $[11,12]$ ).

The applicability of a collisional approximation can be characterized by the two parameters, $\varepsilon=\rho_{i} \lambda_{j} / a^{2}$, and $\mu \equiv \lambda_{i} / a E$, which must be small to have the wave frequency smaller than the ion collision frequency, and mean free path shorter than the parallel wave length ( $a$ is the plasma radius, $\lambda_{i}$ is the ion mean free path, $\rho_{i}$ is the ion gyroradius). We use also derived parameters $M_{0} \equiv 8 \mu^{2} / \varepsilon^{2} \beta$ and $s=a / \rho_{i} \equiv \mu E / \varepsilon$ 
(TABLE I). We assume that the unperturbed state corresponds to equal electron and ion temperatures, but, in perturbations, the electron-ion energy exchange is negligible and, even for the smallest parallel (to the magnetic field) wave number, $k_{z} \sim 1 / E a$, the parallel electron thermal conductivity keeps the electron temperature uniform along the (perturbed) field lines.

Dispersion relations for a number of cases of interest for MTF can be found in [13], which contains a wealth of information on drift instabilitites. As shown in [12], the instability is strongest for the smallest possible parallel wave number compatible with the size of the system. Therefore, we take $k_{z}=1 / E a$. After that, we look for a maximum of Im $\omega / k_{y}{ }^{2}$ over $k_{y}$ which (maximum) we use as a measure of the diffusion coefficient. For large betas, $\beta \gg 1$, the result can be presented in a universal form, as shown in Fig. 1 where $\xi \equiv \partial \ln B / \partial \ln n$. In MTF systems normally $\xi>0$. For the numerical parameters of TABLE I, rows 1 and 2 , even a maximum (over $\xi$ ) value of $D$ is several times less than $D_{B o h m}$.Velocity shear (Sec. 4) may have additional stabilizing effect on drift waves.

In the case of a configuration of the type of a diffuse pinch, (topologically equivalent to the so called MAGO

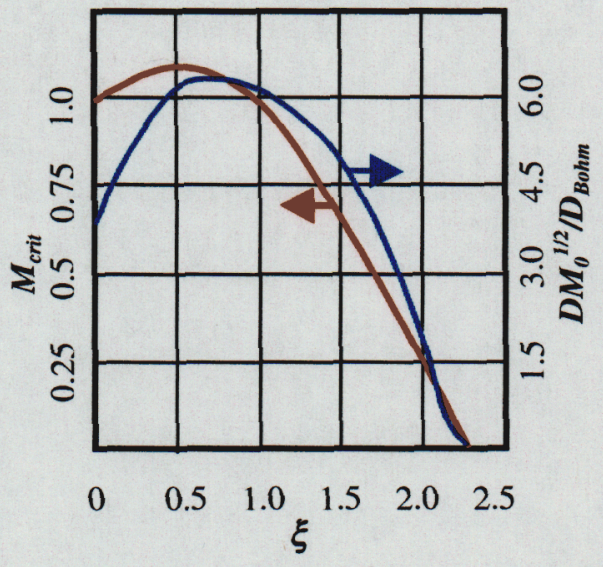

Fig.1 Normalized diffusion coefficient and the parameter $M$; $k_{y}$ of perturbations responsible for transport is $k_{y}=\left(M_{\delta} / M\right)^{1 / 2} / z$. configuration [14]), where only the azimuthal magnetic field is present, there exists a special mode of purely axisymmetric perturbations (analogous to a mode with $k_{z}=0$ ). For this mode effects of the magnetic field line curvature are dominant [13], and the stability criterion is identical to the MHD stability criterion [15]. In particular, in the case of a uniform pressure the plasma is universally stable.

We considered also a high-frequency current-driven lower-hybrid instability [e.g., 16,17] that is thought [18] to be at least partly responsible for the magnetic flux loss in the earlier FRC experiments with $\beta \sim 1$ weakly collisional plasma. This instability depends on the ratio $u / \mathrm{v}_{\mathrm{Ti}}, u=j / e n$. One can estimate it as $u / \mathrm{v}_{T i} \sim \rho_{i} / a \beta=\varepsilon / \mu \beta E$. The effective electron-ion collision frequency is [17]: $v_{\text {eff }} \sim \sqrt{\omega_{C i} \omega_{C e}}\left(u / v_{T e}\right)^{2}$. As $v_{\text {eff }} / v_{e i}=\varepsilon / \beta^{2}$, and $\varepsilon / \beta^{2}<<1$ for the lines 1-3 in TABLE 1 , one sees that the LH instability is unimportant in dense MTF systems.

\section{Evaluation of the role of fusion alpha particles}

MTF systems generally favor lower plasma temperatures than reactors based on standard MFE systems (7-8 keV instead of 20-30 keV), meaning much lower relative density and pressure of the slowing-down the $\alpha$-particles (by a factor of 10-100 less than in conventional systems [19]). Accordingly, in MTF systems, the $\alpha$-particles pressure leads only to small corrections to MHD equilibrium and stability; kinetic instabilities are stabilized by strong plasma collisionality. We conclude that there is no significant adverse effect due to alpha particles in MTF systems.

A magnitude of a favorable effect of $\alpha$-particles (a direct plasma heating) depends on the ratio of the slowing-down time to the residence time of $\alpha$-particles. In the case of a diffuse pinch topology, it has been shown by direct numerical simulation that for the field-times-radius parameter $B R>1 \mathrm{Tm}$ (teslameters) the energy deposition in the plasma is substantially increased over the zero field value. The increase is dependent on field geometry and on the field gradients.

Results for a static azimuthal field having two very different gradients and for a uniform azimuthal field have been used to deduce an approximate dependence on the field gradient of the fraction of the alpha particle energy deposited within a spherical target, 


$$
f_{\alpha}=\rho\left[C(B R)^{2}+R\right] /\left\{\rho\left[C(B R)^{2}+R\right]+D\right\} .
$$

The constant $C$ depends on the plasma temperature, the field gradient length, and the target radius. Using the constant $D=0.2 \mathrm{~g} / \mathrm{cm}^{2}$ [20] and fractional deposition at a single point $(B R=1$ $\mathrm{Tm}$ and $\rho R=0.01 \mathrm{gm} / \mathrm{cm}^{2}$ ), one finds that $C=0.117 \mathrm{~T}^{-2} \mathrm{~m}^{-1}$. The figure 2 shows the agreement of this approximation (bold dashed lines) with particle tracking calculations for two cases $(r \mathrm{R}=0.01$ and $0.001 \mathrm{gm} / \mathrm{cm} 2)$.

\section{Wall confinement.}

In some versions of MTF (for more detail see [2]),

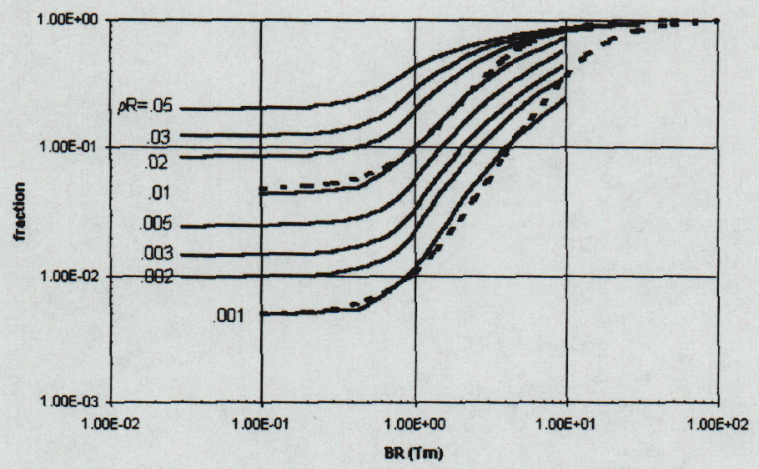

Fig. 2. Comparison of approximation (dashed) in equation against numerical calculations for field due to uniform $\mathrm{j}_{\mathrm{z}}$.

the plasma pressure in the compressed state significantly exceeds the magnetic pressure inside the plasma. Mechanical equilibrium is then provided by transferring the plasma pressure to the wall [5]. [There exist also other versions of MTF, where plasma is separated from the wall by a large vacuum gap $[21,22]$.] The pressure may be transferred to the wall either directly, as a gaseous pressure, or via the magnetic pressure, through a cushion of a strong magnetic field formed near the wall because of cooling and compression of a near-wall plasma $[23,24]$.

We present a series of $1 \mathrm{D}$ cylindrical $\left(B=B_{z}(r)\right.$ only) simulations describing the behavior of a plasma in direct contact with the liner. A programmed, nearly self-similar radial and axial convergence [2] is applied that mocks up the dynamics of self-consistent liner motion. The trajectory is given by: $r=r_{0} f(t) ; f(t)=\left[1-\alpha\left(t / t_{\text {imp }}\right)+0.5 \alpha\left(t / t_{\text {imp }}\right)^{2}\right]^{k}, \alpha=2\left(C^{1 / k}-1\right) / C^{1 / k}$, with $C$ being the radial convergence, taken as $C=10$, and $k=0.5$. For the initial plasma parameters, we choose, $n=10^{18} \mathrm{~cm}^{-3}$, $T=100 \mathrm{eV}, B=100 \mathrm{kG}, r_{0}=1 \mathrm{~cm}$. The velocity source corresponding to this trajectory is applied to the outside of a $500 \mathrm{~mm}$ thick Al liner, with initial velocity of $5 \times 10^{5} \mathrm{~cm} / \mathrm{sec}$. The source is corrected for liner thickening as the material converges so that the liner interior approximately follows the desired trajectory.

Braginski equations are used. Important new ingredients of this set of simulations are a careful analysis of radiative losses from the dense sheath that forms near the wall, modeled with multi-group diffusion, and a detailed characterization of the transition between the plasma and the liner, including a realistic conductivity model [25] and equation of state.

It was shown in [26] that, for the 1D homologous compression, balancing the radiative losses near the wall by a heat flux into the radiating region is possible only if the integral $\int_{T_{\text {wall }}}^{T_{\text {ceper }}}\left(Q_{\text {compr }}-Q_{\text {rad }}\right) \kappa d T$ is positive (here $Q_{\text {compr }}$ and $Q_{r a d}$ are volume densities of the $P d V$ heating and radiative losses, respectively, and $k$ is plasma thermal conductivity). In the case of a uniform magnetic field and classical transport coefficients, this yields the following expression for the minimum implosion velocity: $\mathrm{v}(\mathrm{cm} / \mathrm{s}) \approx 6.9\left[\beta n\left(\mathrm{~cm}^{-3}\right) T(\mathrm{eV})\right]^{1 / 5}$. For the plasma parameters mentioned in the first two lines of Table 1 this yields an acceptable implosion velocity $10^{6} \mathrm{~cm} / \mathrm{s}$. In addition, in systems with a modest $b$, formation of a high-field layer where $k$ decreases and density ceases to increase leads to a suppression of the radiative term in the aforementioned integral. Another factor that may prevent the radiatiove collapse from

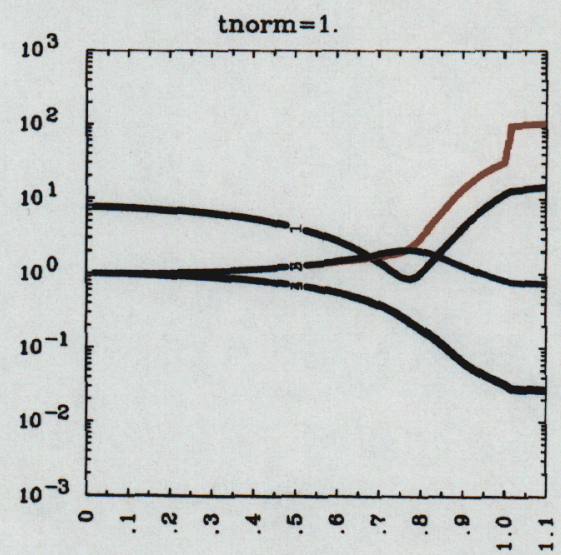

Fig. 3 Normalized density $(\rho(R) / \rho(R=0)$ in red), temperature $(T(R) / T(R=0)$ in dark blue) and magnetic field $(B(R) / B(R=0)$ in light blue) are plotted vs. normalized radius $\left(R / R_{\text {fuel }}\right)$ near peak compression. The plasma beta $\left(8 \pi \mathrm{P} / \mathrm{B}^{2}\right)$ is plotted in black. Poor electrical conductivity of the wall material near the DT plasma edge leads to induction of current and a minimum of beta within the DT plasma. 
happening, is a cooling flow that establishes in the bulk plasma [23].

The solution of this non-steady state problem obtained in our numerical simulations revealed the following interesting features: although a cushion of strong magnetic field is indeed formed near the wall, the pressure is transferred to the wall material mostly as a gas-kinetic pressure as seen in Fig.3; the thickness of the evaporated and partly ionized wall material is comparable to the thickness of the high magnetic field region; The resulting $n t$ for the simulation is $6.5 \times 10^{13}$, with $t$ taken as the fuel stored energy divided by the loss rate at peak compression.

Another new result is related to that we have evaluated the plasma shear flow in the $y$ direction generated in the course of a compression (with $x$ being the radial direction, and $z$ being the direction of the magnetic field). The source term for this motion is a gyroviscosity. Taking into account the enhancement of the azimuthal shear flow in the course of implosion, one can evaluate $\mathrm{v}_{\mathrm{y}}$ as $\zeta\left(\rho_{i} / a\right) \mathrm{v}_{T i}$, with $\zeta \sim 2-3$. The shear flow velocity exceeds phase velocity of drift waves and may further reduce the anomalous transport. It may also have a stabilizing effect on the gross MHD stability [27].

\section{Equilibrium and convection of the plasma configuration of the type of a diffuse pinch}

As a first step in the analysis of $2 \mathrm{D}$ features of the plasma behavior, we consider a configuration of the type of an inverse pinch, i.e., a system where plasma with initial embedded magnetic field is compressed against an outer wall. An experiment to study the rate of heat loss by conduction to the cold boundaries has been proposed at the University of Nevada, Reno. The proposed configuration is being examined using the 2D MHD code MHRDR [28].

Calculations have been done in both $1 \mathrm{D}$ and 2D using Braginskii coefficients, a single-temperature fluid, and Ohm's law in the form $E+V \times B=\eta J$ [29]. In a chamber $40-\mathrm{cm}$ long, with 2 -cm radius inner conductor surrounded by an insulator and $10-\mathrm{cm}$ radius outer conductor (Fig 4), the Zebra pulsed power supply at the Nevada Terawatt Facility would generate $0.5-1$ MA rising in $100-200$ ns. An outwardly accelerated current sheet starts at the inner insulating surface, and collides with the outer conductor. Radial kinetic energy is

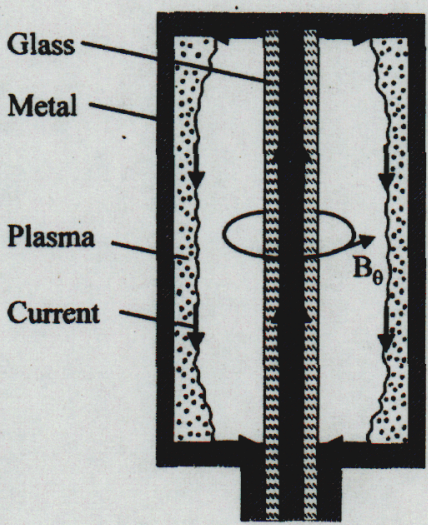

Fig. 4. Geometry of inverse pinch to heat and hold plasma against a room-temperature conductor. mostly dissipated within 1 or $2 \mu$ s giving $\mathrm{T}>100 \mathrm{eV}$ when using an initial fill of 5-50 mtorr deuterium. The hot plasma is held against the outer conducting boundary and cools in about $10 \mu$ s through classical cross-field thermal conduction. Radial magneto-acoustic oscillations are excited that damp more slowly and persist for about $5 \mu \mathrm{s}$, a time comparable to the cooling time.
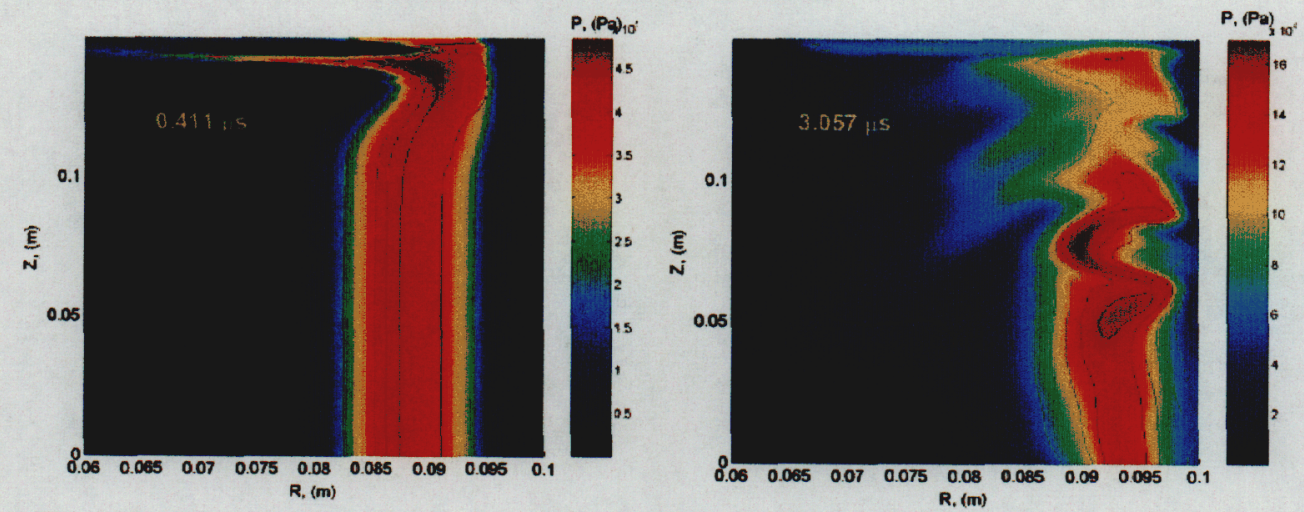

Fig. 5. Pressure contours in shown in color and enclosed current contours (value of $r B_{q}$ ) shown with lines.

An axisymmetric plasma equilibrium in such a system is possible only if the contours of the plasma pressure $p=$ const are nested cylinders (Cf. [30]). As plasma at the ends is in contact with cold electrodes, the plasma pressure necessarily becomes dependent on $z$, the equilibrium condition breaks down, and plasma convection ensues The contact of the plasma with the cold wall during the acceleration phase may give rise to an instability [31] related to Rayleigh-Taylor mode and called in [31] "wall instability." 
In the present calculations, kinetic energy associated with convection grows and then saturates at a low level (a few percent) compared with the thermal energy.

In calculations, sometimes an approximate equilibrium is observed in which radial current flow crossed with the azimuthal field approximately balances pressure gradients in the axial direction. An example of this quasi equilibrium is seen in Fig. 5, where pressure contours in color are seen to be located close to the same places in space where enclosed current contours exist. Structures of the type seen in Fig. 5 are seen to persist for times comparable to the $10-\mu$ s thermal cooling time.

\section{Summary}

We have demonstrated that microturbulence and associated anomalous transport do not impose serious constraints on the performance of the MTF systems. Equally, confinement deterioration caused by the fusion $\alpha$-particles is insignificant. Wall confinement at moderate betas $(\beta \sim 10)$ is not accompanied by catastrophic radiative losses from the dense plasma near the wall. The proposed UN-Reno inverse pinch experiment would allow studying issues of MHD equilibrium, stability, turbulence, and heat loss in curved magnetic field.

This work was performed for the U.S. DoE by UC LLNL under Contract \# W-7405-Eng-48, by UC LANL under Contract \# W-7405-Eng-36, by UNR under Cooperative Agreement DE-FC0801NV14050, and by GA under Grant DE-FG03-95ER54309.

\section{References}

1. R.C. Kirkpatrick, I.R. Lindemuth, M.S. Ward. Fus. Techn., 27, 201 (1995).

2. R.P. Drake et al. Proc. 16th Symposium on Fusion Engineering, Sept. 30-Oct. 5, 1995, v. 1, p. 97; Fus. Techn., 30, $310,1996$.

3. R.E. Siemon, I.R. Lindemuth, K.F. Schoenberg. Comments on Plasma Phys. and Contr. Fus. 18, 363 (1999).

4. D.D. Ryutov, R.E. Siemon. Comments on Modern Phys, 2, \# 5, 185, 2001.

5. G.I. Budker. In: Proc. 6th Europ. Conf. on Controlled Fusion and Plasma Phys., Moscow, 1973, v. 2, p. 136.

6. D.C. Barnes et al. Paper TH/4-5, this Conference.

7. P.B.Parks. Nucl. Fus., 39, 747, 1999; Y.A.Omelchenko, M.J.Schaffer, P.B.Parks.Phys. Plasmas, 8,4463, 2001.

8. D.C. Barnes. Phys. Plasmas. 8, 4856, 2001; 8, 4864, 2001; 9, 560, 2002.

9. E.V. Belova, et al. Phys. Plasmas. 7, 4996, 2000; 8, 1267, 2001.

10. J.M. Taccetti, et al. Bull. Am. Phys. Soc, 45, 247, 2000.

11. A.El Nadi, M.N.Rosenbluth. Phys.Fluids, 16, 2036, 1973; A.Y.Aydemir et al. Phys. Fluids, 30, 3083 (1987).

12. D.D. Ryutov. Phys. Plasmas, 9, 4085, 2002.

13. A.B. Mikhailovskii. "Electromagnetic Instabilities in an Inhomogeneous Plasma" (IoP, Bristol, 1992).

14. I.R. Lindemuth, et al. Phys. Rev. Lett. 75, 1953, 1995.

15. B.B.Kadomtsev. In:Reviews of Plasma Phys. (M.A.Leontovich, Ed.), v.2,p.153, Consult. Bereau, NY, 1966.

16. R.C. Davidson, N.T. Gladd. Phys. Fluids, 18, 1327, 1975.

17. J.F. Drake, et al. Phys. Fluids, 27, 1148, 1984.

18. Tuszhewski, M. Nucl. Fus., 28, 2033 (1988).

19. D.D. Ryutov. Fusion Sci. and Techn., 41, 88, 2002.

20. J. Lindl. Phys. Plasmas, 2, 3933 (1995).

21. P. Turchi. In: Proc. 3rd Intern. Conf. on Megagauss Magnetic Field Generation and Related Topics, Moscow, Nauka Publ. House, 1984, p. 184.

22. T.K. Fowler, LLNL report UCRL-ID-133884 (1999).

23. P.Z. Chebotaev et al, Proc. of the $6^{\text {th }}$ Europ. Conf. on Contr. Fus. And Plasma Phys., Moscow, 1973, v. 1 , p.411.G.E.Vekshtein, et al. Appl. Mech. Techn. Phys., No 6, p.3 (1974) - translated by Plenum 1976.; G.E. Vekstein, G.E. In: "Reviews of Plasma Physics," B.B. Kadomtsev - Ed., Consult. Bureau, New York, 1990.

24. R.E. Waltz. Nucl. Fus., 18, 901, 1978.

25. M. Desjarlais. Contr. Plasma Phys., 41, 267 (2001)

26. P. B. Parks, unpublished, 1998.

27.U. Shumlak, C.W. Hartman. Phys. Rev. Lett. 75, 3285, 1995.

28. I. R. Lindemuth, LLNL Report UCRL-52492, 1979.

29.I. R. Lindemuth, et al., Proceedings $29^{\text {th }}$ IEEE International Conf. on Plasma Sci., Banff, Canada (2002).

30. V.D. Shafranov. In: "Rev. of Plasma Phys." (M.A.Leontovich - Ed.), v.2,p.103, Consult. Bureau, NY 1966.

31. T. W. Hussey, N. F. Roderick, and D. A. Kloc, J. Appl. Phys. 51, 1452 (1980). 